\title{
Periprosthetic Fractures Following Total Knee Arthroplasty
}

\author{
Jae Doo Yoo, MD and Nam Ki Kim, MD \\ Department of Orthopedic Surgery, Ewha Womans University Mokdong Hospital, Seoul, Korea
}

\begin{abstract}
Periprosthetic fractures after total knee arthroplasty may occur in any part of the femur, tibia and patella, and the most common pattern involves the supracondylar area of the distal femur. Supracondylar periprosthetic fractures frequently occur above a well-fixed prosthesis, and risk factors include anterior femoral cortical notching and use of the rotational constrained implant. Periprosthetic tibial fractures are frequently associated with loose components and malalignment or malposition of implants. Fractures of the patella are much less common and associated with rheumatoid arthritis, use of steroid, osteonecrosis and malalignment of implants. Most patients with periprosthetic fractures around the knee are the elderly with poor bone quality. There are many difficulties and increased risk of nonunion after treatment because reduction and internal fixation is interfered with by preexisting prosthesis and bone cement. Additionally, previous soft tissue injury is another disadvantageous condition for bone healing. Many authors reported good clinical outcomes after non-operative treatment of undisplaced or minimally displaced periprosthetic fractures; however, open reduction or revision arthroplasty was required in displaced fractures or fractures with unstable prosthesis. Periprosthetic fractures around the knee should be prevented by appropriate technique during total knee arthroplasty. Nevertheless, if a periprosthetic fracture occurs, an appropriate treatment method should be selected considering the stability of the prosthesis, displacement of fracture and bone quality.
\end{abstract}

Keywords: Knee, Arthroplasty, Periprosthetic fracture, Internal fixation, Revision total knee arthroplasty

\section{Introduction}

Improvements in quality of life and life expectancy have resulted in substantial increases in the incidence of total knee arthroplasty (TKA) and thus the likelihood of postoperative complications. The risk of periprosthetic fracture following TKA is particularly high because most of the TKA patients are advanced in age and have osteopenia ${ }^{1,2)}$. Periprosthetic fractures can occur in the femur, tibia or patella, affecting the areas within $15 \mathrm{~cm}$ from the joint surface or within $5 \mathrm{~cm}$ from the intramedullary stem ${ }^{1-3)}$. The femur is the most frequent fracture site followed by the tibia and

Received April 3, 2014; Revised (1st) May 24, 2014; (2nd) June 29, 2014; Accepted July 2, 2014

Correspondence to: Jae Doo Yoo, MD

Department of Orthopedic Surgery, Ewha Womans University Mokdong Hospital, 1071 Anyangcheon-ro, Yangcheon-gu, Seoul 158-710, Korea Tel: +82-2-2650-6142, Fax: +82-2-2650-0349

E-mail: koreanknee@gmail.com

This is an Open Access article distributed under the terms of the Creative Commons Attribution Non-Commercial License (http://creativecommons.org/licenses/by-nc/3.0/) which permits unrestricted non-commercial use, distribution, and reproduction in any medium, provided the original work is properly cited. patella $^{1,2)}$. Compared to other fractures, periprosthetic fractures are more challenging to the surgeon in terms of fracture treatment and patient recovery. Poor bone stock, preexisting implant and bone cement may impede fracture reduction and fixation, predisposing to nonunion or malunion ${ }^{1,4,5)}$. Revision TKA may be required due to implant instability or soft tissue adhesion, or vascular damage may delay wound healing and increase the risks of infection and necrosis ${ }^{2,4)}$. In addition, combined medical conditions often disrupt postoperative recovery, rehabilitation, and ambulation. Nevertheless, satisfactory clinical outcomes can be obtained with proper selection of fixation devices and surgical techniques. Therefore, accurate diagnosis and appropriate intervention are of utmost importance in the treatment of periprosthetic fractures.

\section{Supracondylar Fractures of the Femur}

\section{Causes and Risk Factors}

Periprosthetic fracture following TKA occurs most frequently in the femur, primarily in the supracondylar area ${ }^{1,2)}$ (Fig. 1). Femoral supracondylar fractures are observed in $0.3 \%-2.5 \%$ of 

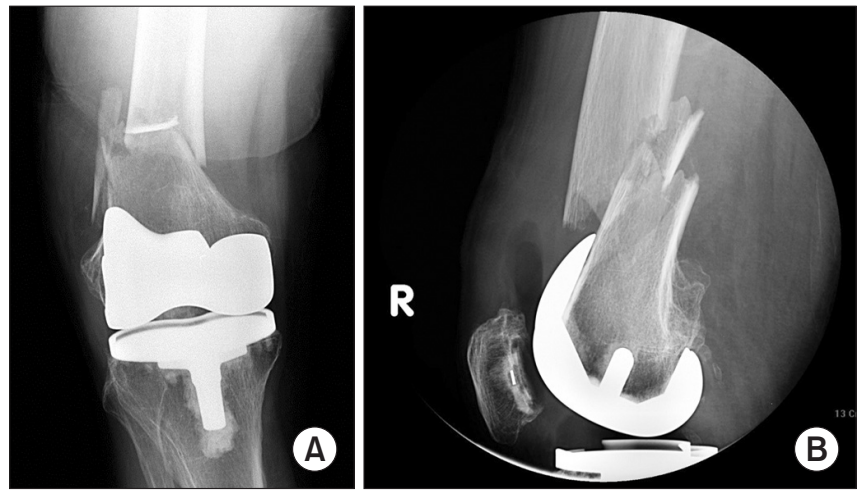

Fig. 1. (A) Anteroposterior radiograph showing a displaced and comminuted supracondylar femoral fracture with osteopenia. (B) Lateral radiograph showing a displaced supracondylar femoral fracture of the same patient.
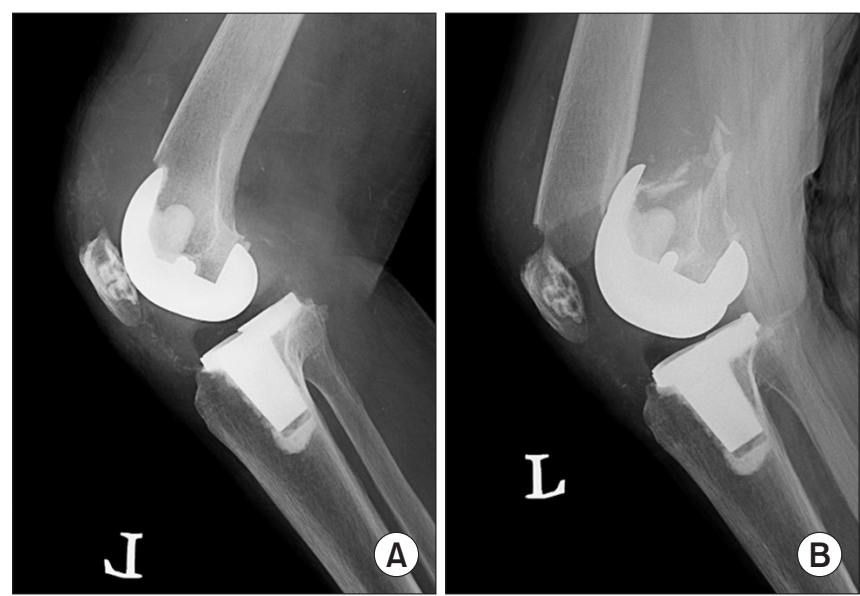

Fig. 2. (A) Lateral radiograph showing a notching of the anterior femoral cortex after total knee arthroplasty. (B) Lateral radiograph at postoperative 2 years showing a displaced supracondylar femoral fracture at the site of cortical notching.

TKA patients mostly within 2 to 4 years after surgery ${ }^{5-7)}$. They are commonly noted in the knee with a well-fixed femoral component mostly after low-energy injuries caused by torsional or compression forces and sometimes after high-energy trauma ${ }^{2,8)}$. Risk factors for femoral supracondylar fractures include anterior femoral notching, mismatch of the elastic modulus between the metal implant and the femoral cortex, rotationally constrained components, osteolysis, delayed bone remodeling due to vascular compromise at the surgical site, knee joint ankylosis, previous revision TKA, prolonged steroid use, osteoporosis, rheumatoid arthritis, advanced age, female gender and neurologic disorders ${ }^{2,3,5}$. Femoral fractures can also occur after navigation-assisted TKA at pinhole sites ${ }^{9}$. Anterior femoral notching refers to deformation of the anterior femoral cortex caused by external forces, which

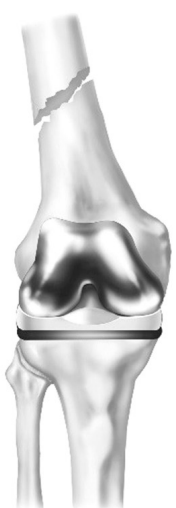

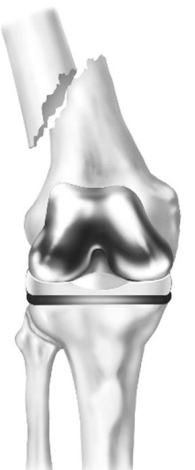

III

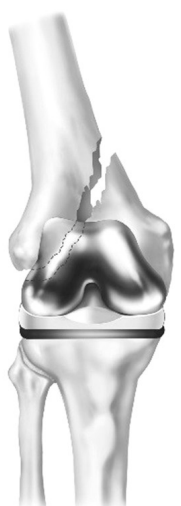

Fig. 3. Classification of supracondylar femoral fractures above total knee arthroplasty described by Rorabeck and Taylor ${ }^{12)}$.

mostly results from excessive resection of the anterior femur during surgery (Fig. 2). Several studies have demonstrated that anterior femoral notching is closely associated with femoral supracondylar fractures: they occurred in $10 \%-46 \%$ of the notched femora $^{3,10)}$. Culp et al. ${ }^{3)}$ reported that a 3-mm anterior cortical notch would result in $30 \%$ reduction in torsional bone strength, predisposing to the risk of femoral fracture. If a fracture is suspected, long-leg radiography should be performed for confirmation. If pain is present without evidence of an obvious fracture or prosthesis loosening, blood tests and joint aspiration should be conducted to test for an infection.

\section{Classification}

The traditional Neer classification has some limitations in that it does not take into account the implant fixation status and bone quality that are crucial in determining treatment strategy ${ }^{11}$. Thus, the classification system proposed by Rorabeck and Taylor ${ }^{12)}$ in 1998 is currently favored by many orthopedists (Fig. 3). The Rorabeck and Taylor ${ }^{12)}$ classification takes into consideration the implant fixation status and displacement of fracture. Type I is assigned to a non-displaced fracture around a well-fixed prosthesis. Type II fractures have $\geq 5 \mathrm{~mm}$ displacement or $\geq 5^{\circ}$ angulation with the prosthesis remaining stable, which are subdivided into type IIA (non-comminuted fractures) and type IIB (comminuted fractures). A type III fracture is accompanied by component loosening/instability and polyethylene wear irrespective of the displacement of fracture fragments. Su et al. ${ }^{13)}$ suggested a classification system that accounts for the height of a fracture line relative to the femoral component: type I fractures are proximal to the femoral component; type II fracture lines originate at the proximal end of the femoral component and partially extend proximally; and type III fractures occur distal to the upper edge 
of the femoral component. A classification system introduced by Kim et al. ${ }^{14)}$ factors in the remaining bone stock, prosthesis fixation status, and reducibility of the fracture (Table 1): type I is defined as a fracture that occurs in the knee with an intact prosthesis and sufficient bone stock, which is subcategorized into type IA that is amenable to conservative treatment and reduction and type IB that requires surgical reduction and internal fixation; type II is assigned if a revision surgery is required due to unstable fixation or malposition of the prosthesis in spite of sufficient bone stock and reducibility; and type III is defined as a severely comminuted facture with poor bone stock.

\section{Treatment}

In order to prevent periprosthetic fracture following TKA, preexisting metal plates or screws implanted for previous fracture treatment should be removed three months in advance if the fracture healed completely. In cases where implant removal and TKA are carried out concurrently, a long-stemmed prosthesis is used so that it can be inserted through the previous fracture site. When a posterior cruciate ligament (PCL)-substituting prosthesis is utilized, care should be taken to avoid eccentric placement of the box cut ${ }^{11}$. If anterior femoral notching occurred intraoperatively, a long-stemmed prosthesis should be implanted to reduce the stress on the anterior femoral cortex, and postoperative weight-bearing should be restricted with use of walking aids or crutches. Nevertheless, if a fracture occurs after TKA, proper treatment should be applied to promote fracture union and joint function recovery. Acceptable alignments after fracture reduction are $<5 \mathrm{~mm}$ translation, $<5^{\circ}-10^{\circ}$ angulation, $<10^{\circ}$ rotational deformity, and $<1 \mathrm{~cm}$ femoral shortening ${ }^{15)}$. In the presence of severe

Table 1. Classification of Supracondylar Fractures of the Distal Femur after Total Knee Arthroplasty Described by Kim et al. ${ }^{14)}$

\begin{tabular}{cll}
\hline Type & & \multicolumn{1}{c}{ Characteristics of fracture } \\
\hline IA & Good bone stock, stable and well postioned prosthesis, non-displaced or easily reducible fracture & Conservative treatment \\
IB & Good bone stock, stable and well postioned prosthesis & Reduction and internal fixation \\
II & Reducible fractures with adequate distal bone, malpositioned or loose component & Revision with a long stem component \\
III & Severely comminuted fractures with inadequate distal bone for fixation & Prosthetic replacement \\
\hline
\end{tabular}

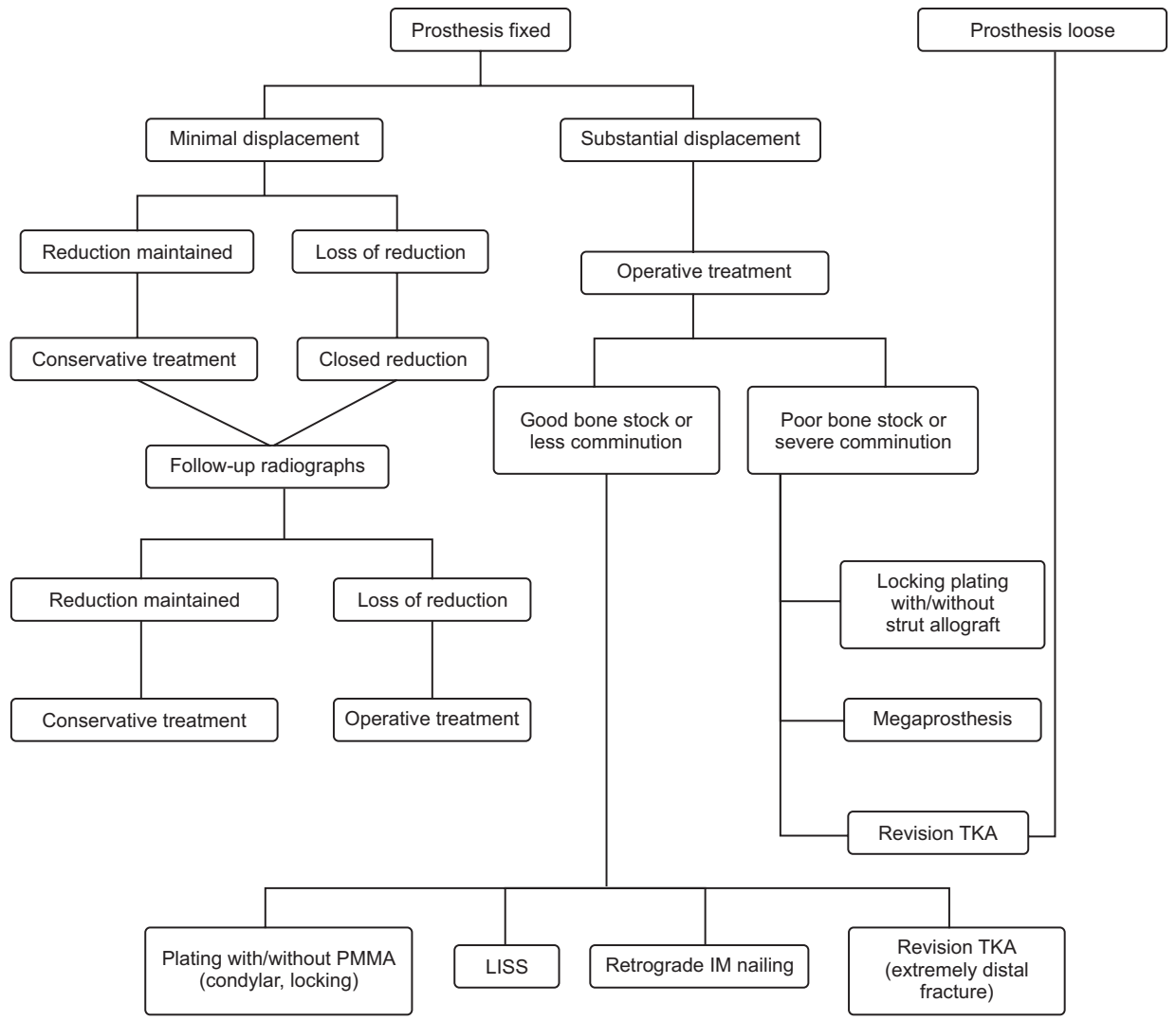

Fig. 4. Algorithm of treatment options for supracondylar femoral fractures above total knee arthroplasty (TKA). PMMA: polymethyl methacrylate, LISS: less invasive stabilization system, IM: intramedullary. 
fracture displacement, comminution or intercondylar extension, it is difficult to expect satisfying treatment outcomes. In addition, the adductor and gastrocnemius muscles may cause malunion associated with varus, flexion and internal rotation deformities of the distal fragment ${ }^{(6)}$. Figgie et al. ${ }^{6}$ reported that progressive radiolucent lines were observed at the bone-cement interface in 9 of the 12 femoral supracondylar fractures that had united with varus malposition of the femoral component. Treatment options according to the type of femoral supracondylar fracture are summarized in Fig. 4.

\section{1) Non-operative treatment}

Non-operative treatment includes skeletal traction, splinting, casting and cast bracing. Currently, skeletal traction is rarely employed due to the risk of pin site infection, nerve palsy, bed sore caused by prolonged immobilization, progression of osteoporosis and knee joint ankylosis. Non-operative treatment is advantageous in that general anesthesia is not necessary and complications caused by hemorrhage and infection are rare. Thus, it can considered as a treatment option for early stage Rorabeck type I fractures. If fracture reduction is determined necessary, closed reduction is followed by $4-6$ weeks of cast immobilization (Fig. 5), during which the alignment of the fracture and implant stability are regularly monitored with biweekly radiographs. When proper knee alignment is maintained, the cast is removed and progressive range of motion exercises are initiated with the knee protected in a hinged brace. If displacement of the fracture or implant instability is suspected during follow-up, the non-operative therapy may need to be replaced by aggressive operative treat-
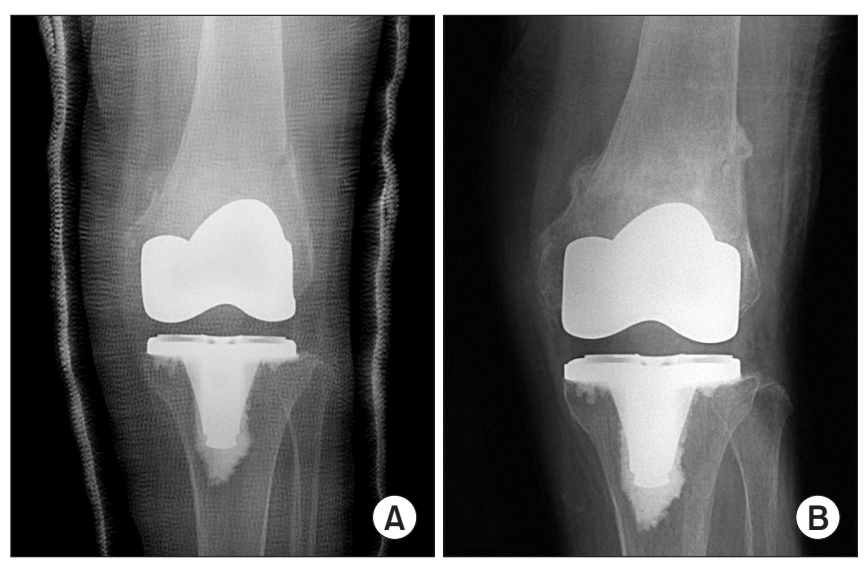

Fig. 5. (A) Anteroposterior radiograph showing a minimally displaced supracondylar femoral fracture treated with cast immobilization. (B) Anteroposterior radiograph at 1 year after non-operative treatment showing radiologic fracture union. ment $^{2,5,15)}$. Chen et al. ${ }^{5)}$ compared the results of non-operative and operative treatments for periprosthetic fractures of the femur in a literature review. The success rate of non-operative treatment for non-displaced fractures was $83 \%$. Regarding the treatment for displaced fractures, there was no significant difference in patient satisfaction between the operative treatment group and non-operative treatment group (61\% vs. $67 \%$ ). In addition, the incidence of complications was higher in the operative treatment group. However, in the presence of severe displacement of the fragment or combined osteoporosis, reduction can be either unsuccessful or difficult to maintain with non-operative methods and the risk of malunion is high ${ }^{2,15}$. In a study by Moran et al. ${ }^{16)}$, malunion occurred in all of the 9 patients after conservative treatment for displaced fracture, whereas 10 of the 15 patients with operative treatment obtained excellent results. Therefore, proper patient selection is essential for the success of non-operative treatment in cases of Rorabeck type II and III fractures, considering the high risk of nonunion and malunion.

\section{2) Operative treatment}

For unstable, displaced fractures, anatomical reduction and rigid fixation obtained by operative treatment allows early range of motion exercises and ambulation ${ }^{2,5}$. Common surgical treatment techniques include external fixation, internal fixation using blade plates, condylar screws, retrograde intramedullary nails or locking plates and revision TKA.

\section{(1) Internal fixation with metal plates}

Internal fixation devices, such as 95-degree angled blade plates, dynamic condylar screws and condylar buttress plates, do not provide stable fixation for comminuted supracondylar fractures with poor bone stock and can cause interference with the femoral component or cement ${ }^{2,12,15}$. Thus, research has been conducted to ensure rigid fixation in the osteopenic bone with use of bone grafting or bone cement augmentation. Healy et al. ${ }^{17)}$ treated 20 Rorabeck type II fractures using blade plates and condylar screw plates, and additionally performed bone grafting in 15 knees with severe osteoporosis. The fractures healed in all knees although revision surgery was required due to loss of reduction in two knees. Presently available locking metal plates are fixed-angle devices designed to have threads that engage with the screw threads ${ }^{18)}$. They ensure more rigid fixation than traditional metal plates for periarticular, comminuted and osteoporotic fractures ${ }^{15,18)}$ (Fig. 6). In the presence of severe comminution in the medial aspect of the femur, dual plating can be considered as an option to prevent varus deformity following collapse of the medial cortex due to 

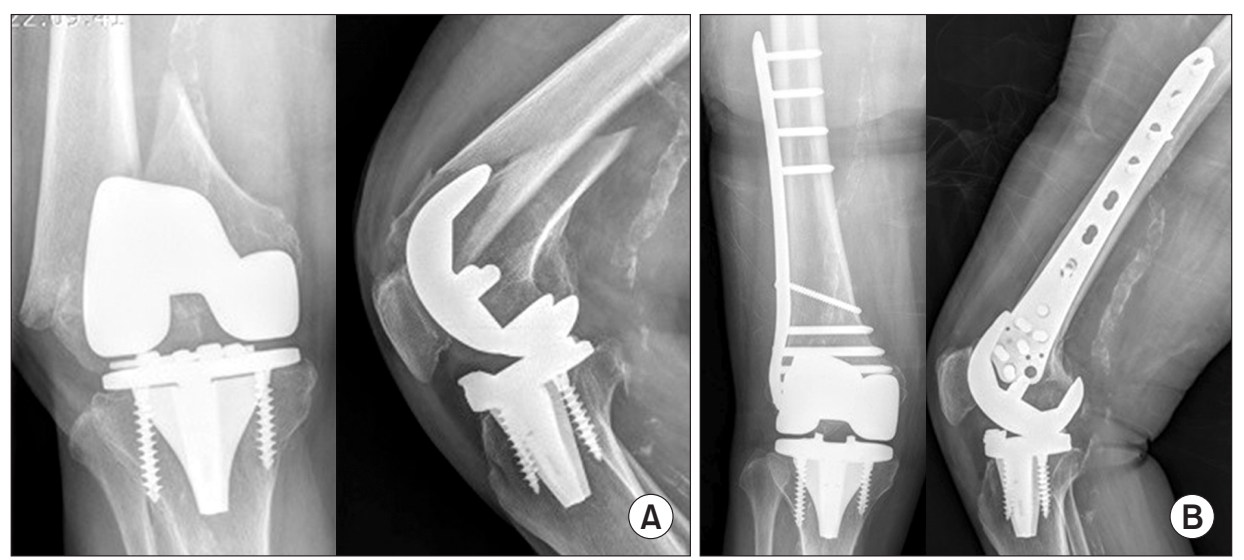

Fig. 6. (A) Anteroposterior and lateral radiographs of a displaced supracondylar femoral fracture following revision total knee arthroplasty. (B) Anteroposterior and lateral radiographs showing anatomical reduction of fracture after open reduction and internal fixation using a locking plate.
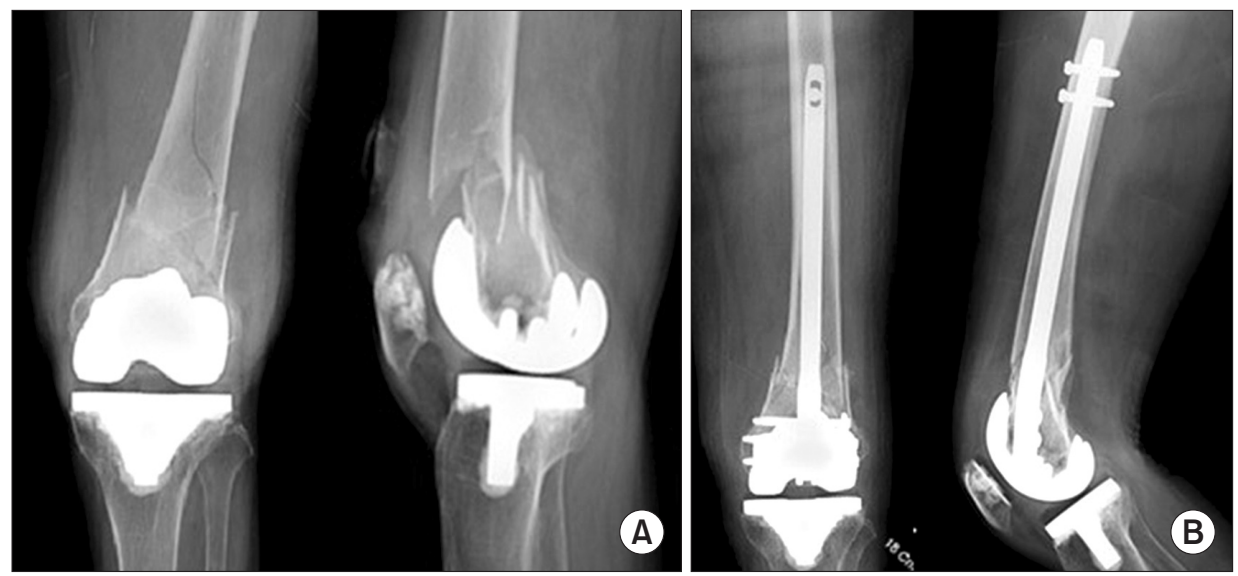

Fig. 7. (A) Anteroposterior and lateral radiographs of a displaced supracondylar femoral fracture following total knee arthroplasty. (B) Anteroposterior and lateral radiographs showing satisfactory fracture reduction after reduction and internal fixation using a retrograde intramedullary nail.

lateral plating alone ${ }^{19)}$. Disadvantages of internal fixation with metal plates include the need for a large surgical excision, extensive soft tissue damage and hemorrhage and the increased risk of nonunion due to damage to the periosteum and blood vessels. Recently, minimally invasive internal fixation using a locking plate (less invasive stabilization system [LISS]) has been introduced. The concept of LISS is to minimize injury to the adjacent soft tissue and periosteum and disruption of blood supply with a minimal incision and stripping during surgery. It promotes rapid bone union with low risk of complications, such as hemorrhage and infection, compared to traditional techniques ${ }^{15,20)}$. Some locking metal plates allow for closed reduction with use of percutaneous screw insertion or a retractor based on the principle of ligamentotaxis ${ }^{20)}$. Kregor et al. ${ }^{21)}$ performed LISS fixation for the treatment of supracondylar fractures and obtained bone union in 36 of 38 knees without any complications.

(2) Retrograde intramedullary nailing

Retrograde intramedullary nailing is recommended for the treatment of periprosthetic fractures of the supracondylar femur because more than two antegrade intramedullary nails cannot be inserted into the distal fragment in most cases and fixation of these nails is not reliable ${ }^{22)}$. Retrograde intramedullary nailing causes less soft tissue damage and hemorrhage than the conventional metal plate fixation. In addition, it is advantageous in obtaining bone union due to the preservation of periosteal blood supply and fracture hematoma. Flexible intramedullary nails that were preferred by the orthopedists in the past can only be considered as an option in cases of mildly displaced fractures due to the risk of lower limb shortening and rotational malunion attributable to the their inherent vulnerability to the compression and torsional forces ${ }^{2}$. Currently, interlocking intramedullary nailing using interlocking screws with higher resistance to axial compression and torsional forces is commonly utilized (Fig. 7). Interlocking intramedullary nailing for Rorabeck type II supracondylar femoral fractures yields high union rates and excellent functional improvement ${ }^{23)}$. Retrograde intramedullary nails should be long enough to reach the level of the lesser trochanter because their passage through the isthmus of the femur prevents 
windshield wiper effect and improves stability ${ }^{24)}$. Hyperextension of the femoral component may be noted in the sagittal plane because reaming and insertion are performed with the knee in flexion position, which is more notable when the insertion site is extremely posterior to the intercondylar notch. According to Pelfort et al. ${ }^{25}$, hyperextension of the femoral component did not significantly affect stability of the prosthesis, bone union and knee joint function. Besides, valgus malalignment of the distal fragment in the coronal plane is often encountered after fracture treatment. To avoid this, it is recommended to use a blocking screw as a guide for proper insertion of the intramedullary nail ${ }^{26)}$. Contraindications to intramedullary nailing include patellar baja, joint ankylosis precluding intramedullary nail insertion, $<11 \mathrm{~mm}$ intercondylar distance or narrow medullary cavity, preexisting intramedullary stem in the proximal femur from previous total hip arthroplasty, severe comminution or extremely distal fracture precluding stable internal fixation and unstable TKA prosthesis $^{24-26)}$. Some PCL-substituting implants are closed box designs in which the metal intercondylar box completely covers the intercondylar notch, allowing insertion of an interamedullary nail after drilling with a diamond-tip metal-cutting burr ${ }^{27}$.

\section{(3) Revision total knee arthroplasty}

Irrespective of the presence of displacement or comminution, a revision TKA should be considered when a fracture is combined with femoral component instability ${ }^{1,2,5}$. Even in the absence of component instability, revision TKA may be necessitated in the following conditions: severe comminution or a fracture in the vicinity of the articular surface precluding internal fixation, failure of other treatments or severe malalignment of the knee prosthe$\operatorname{sis}^{2-5)}$.

During revision TKA, a long-stemmed femoral component is inserted through the fracture site into the proximal medullary cavity of the femur ${ }^{1,3,5,6)}$. Although cemented femoral components are commonly used, if fracture site stability cannot be obtained with use of a long-stemmed femoral component alone, strut allograft or cerclage wiring should be additionally performed to ensure stability of the fracture site and femoral component ${ }^{1,6)}$. If nonunion occurs either due to the deficiency of bone tissue that supports the femoral component in cases of severe comminution or bone loss or due to other internal fixation failure, distal femoral replacement using an allograft tumor prosthesis may be considered as a limb salvage procedure to preserve minimum knee function and maintain the leg length ${ }^{28}$. Freedman et al. ${ }^{28)}$ reported that distal femoral replacement performed using a tumor megaprosthesis due to severe nonunion and comminution following internal fixation of periprosthetic supracondylar fractures resulted in early ambulation and $100^{\circ}$ of range of motion in 4 of the total 5 knees.

\section{Periprosthetic Fractures of the Tibia}

\section{Causes and Risk Factors}

The prevalence of periprosthetic fractures of the tibia is $0.4 \%-$ $1.7 \%$, which is relatively low compared to that of the femur ${ }^{29,30}$. It can happen at any stage of surgery, especially during skeletal retraction, trial component insertion, reaming of the tibia, cementation, implant insertion, polyethylene insert placement and removal of the preexisting prosthesis in revision surgery ${ }^{31,32)}$. Some studies associated the preoperative neutral/valgus alignment with tibial fractures, and tibial shaft fractures were attributed to tibial tubercle osteotomy $y^{32)}$. Most postoperative fractures result from acute trauma, but stress fractures without any trauma are also encountered ${ }^{29)}$. Tibial fractures are often accompanied by component loosening or instability and sometimes by component malalignment, malposition and joint instability ${ }^{2,30,32)}$. Rand and Coventry ${ }^{29)}$ noted malalignment of the knee joint or malposition of the tibial component in all of the patients $(n=15)$ with stress fractures of the tibia following TKA. Lotke and Ecker ${ }^{33)}$ suggested that varus fixation of the tibial component was correlated with medial plateau fracture. During TKA, care should be taken not to place the tibial component in the excessively lateral aspect of the knee in patients with a history of high tibial osteotomy in order to avoid passage of the tibial component stem through the lateral cortex of the tibia ${ }^{11)}$.

\section{Classification}

According to the most widely used classification system suggested by Felix et al. ${ }^{30)}$, periprosthetic fractures of the tibia can be categorized into four types (I-IV) and three subtypes (A, B, C) for each type based on the anatomical location of the fracture and the status of the tibial component fixation ${ }^{30)}$ (Fig. 8). Type I fractures are located at the tibial plateau, type II fractures occur inferior to the tibial plateau adjacent to the prosthetic stem, type III fractures occur distal to the tibial stem and type IV fractures involve the tibial tubercle. Type A is assigned to a fracture with a stable prosthesis on radiographs, type $B$ is defined as fractures with radiographic evidence of component loosening and type $\mathrm{C}$ refers to intraoperative fractures.

\section{Treatment}

Treatment decisions are dependent on the fracture site and the 


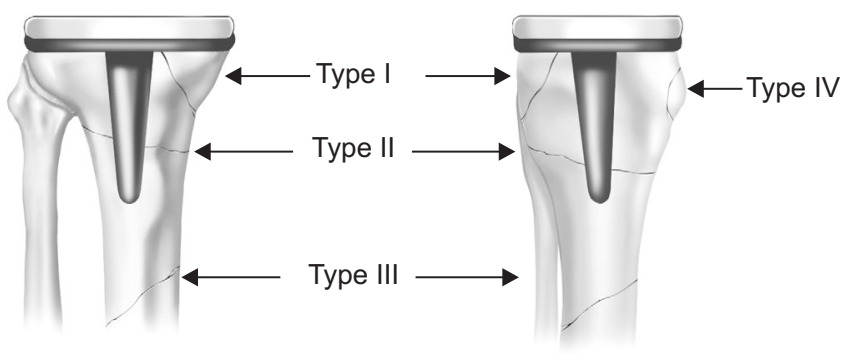

Anteroposterior

Lateral

Fig. 8. Classification of periprosthetic tibial fractures described by Felix et al. $^{30)}$.

status of the prosthesis: if the tibial prosthesis is intact, the fracture can be treated with standard treatment approaches ${ }^{30)}$. In the knee with a normal component alignment and stable fixation and minor displacement of the fracture, closed reduction and cast immobilization would result in satisfying results ${ }^{30)}$. However, a severely displaced, unstable fracture necessitates open reduction and internal fixation even in the presence of stable component fixation. In such a case, metal plates are commonly used for internal fixation because the preexisting tibial component precludes insertion of intramedullary nails ${ }^{30,31)}$. Even in the knee with poor bone stock, locking plates with several locking screws inserted around the tibial stem allow rigid fixation. In cases of intraoperative fractures, treatment decisions are based on the status and site of the fracture. For unstable type IC fractures, screw fixation of bone fragments is followed by insertion of a long-stemmed tibial component through the fracture site to the tibial medullary cavity. Type IIC fractures are treated using a long-stemmed tibial component and bone graft for bone defects at the fracture site. Type IIIC fractures are managed with either internal fixation or conservative treatment based on the fracture site and pat$\operatorname{tern}^{31)}$. Type IV fractures that involve the tibial tubercle should be treated with care to avoid disruption of the extensor mechanism. Felix et al. ${ }^{30)}$ obtained bone union without any complication in 2 type IVA fractures after cast immobilization with knee extension in one case and screw fixation in the other case. Hanssen and Stuart $^{34)}$ suggested using polypropylene mesh tape or semitendinosus rerouting for fixation of tibial bone fragments in cases of type IV fractures. If tibial component instability is noted, revision TKA should be considered as an option ${ }^{8,29,30)}$. In revision TKA, the tibial component of choice should be equipped with a longer than normal stem so that it can be fixed to the tibial shaft after being passed through the fracture site in the proximal tibia ${ }^{2,29,30)}$. After tibial component fixation, additional internal fixation may be required for the remaining fixable unstable bone fragments.
For any bone defects in the proximal tibia, metal augmentation should be performed to obtain implant stability. Metal augmentation and thick polyethylene insertion can be effective for treating $\leq 5 \mathrm{~cm}$ deep bone defects; however, severe bone defects or comminuted fractures should be managed with either strut allograft or tumor megaprosthesis ${ }^{34)}$.

\section{Periprosthetic Fractures of the Patella}

\section{Causes and Risk Factors}

Periprosthetic fracture of the patella is an uncommon complication of TKA. The incidence of patellar fractures has been reported as $0.2 \%-21 \%$ in the patella-resurfaced knee and $0.05 \%$ in the non-resurfaced $\mathrm{knee}^{35,36)}$. Patellar fractures are more frequent in males than females unlike femoral and tibial fractures; although the exact reason has not been elucidated, it has been understood that higher level of activities and weights results in greater extension of the knee and thus the higher male susceptibility ${ }^{37)}$. Patellar fractures can occur due to direct trauma or fatigue ${ }^{35,36)}$. The identified risk factors include rheumatoid arthritis, prolonged steroid use, patellar necrosis, malalignment of the lower limb or knee prosthesis and PCL-substituting implant, and certain types of patellar prostheses and surgical techniques have been associated with the fracture ${ }^{35-37)}$. Malalignment of the femoral component imposes increased eccentric load on the femorotibial joint and causes subluxation of the patella, eventually resulting in a patellar fracture $^{38)}$. Excessive stripping of soft tissue adjacent to the patella may cause disruption of osseous blood supply, increasing the risk of fracture resulting from osteonecrosis ${ }^{35-37,39)}$. Asymmetric resection of the patella increases mechanical strain on the patella especially when the subchondral bone or the lateral articular surface is included in the resection. Conversely, insufficient resection results in increased patellar-implant thickness, causing greater patellofemoral joint reaction force and excessive traction on the extensor mechanism ${ }^{35-37)}$. In addition, excessive patellar eversion may cause a fracture or a rupture of the patellar tendon; thus, it is advised to perform distal femoral resection prior to eversion when it is difficult to perform ${ }^{11)}$. The implant design also has an influence on the risk of fracture: implants with a large central peg causes greater anterior patellar strain than those with small peripheral pegs, resulting in increased fracture risks ${ }^{40)}$. Other predisposing factors include osteonecrosis caused by heat generated by bone cement polymerization and compression forces generated during repeated knee flexion of $\geq 95^{\circ 36,37)}$. Thus, it is recommended to promote restoration of the original patellar thickness after proper and symmetrical resection of the patella and implant 
insertion $^{35,36)}$. Peripheral peg designs are more desirable than central peg designs to reduce the risk of failure ${ }^{40}$. Besides, care should be taken to prevent osteonecrosis by preserving peripatellar soft tissue as much as possible and to protect the lateral superior genicular artery from injury during lateral retinacular release $\mathrm{s}^{36,39)}$.

\section{Classification}

Goldberg et al. ${ }^{35)}$ categorized patellar fractures into four types according to the fracture configuration, stability of the patellar component, and integrity of the extensor mechanism. Goldberg type I fractures are located in the periphery of the patella and do not involve the patellar component and the extensor mechanism. Type II fractures disrupt the implant-bone composite or the extensor mechanism. Type III fractures involve the inferior pole of the patella, which are subcategorized into type IIIA with patellar ligament rupture and type IIIB without patellar ligament rupture. Type IV fractures refer to patellar fractures accompanied by patellofemoral dislocation.

\section{Treatment}

Conservative treatment can be considered as a treatment option in the absence of disruption of the extensor mechanism and patellar component instability ${ }^{35,37)}$. Ortiguera and Berry ${ }^{37)}$ reported that bone union and restoration of knee function were achieved with conservative treatment in 37 out of 38 knees with mildly displaced fractures and stable component fixation. In the absence of established treatment protocols for the knee with mild disruption of the extensor mechanism without component instability, complications rates after internal fixation can be as high as $50 \%{ }^{37}$. For treatment of severely comminuted fractures, some studies recommended nonsurgical treatment or removal of small bone fragments and attachment of the patellar tendon or quadriceps femoris tendon to the bon $\mathrm{e}^{2,37}$. If implant instability is present, the treatment decision should be based on the remaining bone stock. In the knee with sufficient bone stock, removal of the preexisting prosthesis is followed by revision TKA or patelletcomy. On the other hand, in the knee with poor bone stock, partial or complete patellectomy is recommended over revision TKA. However, given the reports on high complication rates of surgical treatment for fractures with patellar component instability, non-operative treatment can be considered in patients with mild symptoms and good knee function. Most of such patients present with no or mild pain, and thus patellar fractures are often discovered accidentally on radiographs and 4-6 weeks of joint immobilization could yield satisfying results ${ }^{2,35,37)}$.

\section{Conflict of Interest}

No potential conflict of interest relevant to this article was reported.

\section{References}

1. Cordeiro EN, Costa RC, Carazzato JG, Silva Jdos S. Periprosthetic fractures in patients with total knee arthroplasties. Clin Orthop Relat Res. 1990;(252):182-9.

2. Dennis DA. Periprosthetic fractures following total knee arthroplasty. J Bone Joint Surg Am. 2001;83:120-30.

3. Culp RW, Schmidt RG, Hanks G, Mak A, Esterhai JL Jr, Heppenstall RB. Supracondylar fracture of the femur following prosthetic knee arthroplasty. Clin Orthop Relat Res. 1987;(222):212-22.

4. Engh GA, Ammeen DJ. Periprosthetic fractures adjacent to total knee implants: treatment and clinical results. Instr Course Lect. 1998;47:437-48.

5. Chen F, Mont MA, Bachner RS. Management of ipsilateral supracondylar femur fractures following total knee arthroplasty. J Arthroplasty. 1994;9:521-6.

6. Figgie MP, Goldberg VM, Figgie HE 3rd, Sobel M. The results of treatment of supracondylar fracture above total knee arthroplasty. J Arthroplasty. 1990;5:267-76.

7. Rorabeck $\mathrm{CH}$, Taylor JW. Periprosthetic fractures of the femur complicating total knee arthroplasty. Orthop Clin North Am. 1999;30:265-77.

8. Scott WN, Insall JN. Insall \& Scott surgery of the knee. 5th ed. Philadelphia, PA: Elsevier; 2012. p799-807.

9. Li CH, Chen TH, Su YP, Shao PC, Lee KS, Chen WM. Periprosthetic femoral supracondylar fracture after total knee arthroplasty with navigation system. J Arthroplasty. 2008;23: 304-7.

10. Ritter MA, Faris PM, Keating EM. Anterior femoral notching and ipsilateral supracondylar femur fracture in total knee arthroplasty. J Arthroplasty. 1988;3:185-7.

11. Backstein D, Safir O, Gross A. Periprosthetic fractures of the knee. J Arthroplasty. 2007;22(4 Suppl 1):45-9.

12. Rorabeck CH, Taylor JW. Classification of periprosthetic fractures complicating total knee arthroplasty. Orthop Clin North Am. 1999;30:209-14.

13. Su ET, DeWal H, Di Cesare PE. Periprosthetic femoral fractures above total knee replacements. J Am Acad Orthop Surg. 2004;12:12-20.

14. Kim KI, Egol KA, Hozack WJ, Parvizi J. Periprosthetic fractures after total knee arthroplasties. Clin Orthop Relat Res. 
2006;446:167-75.

15. DiGioia AM 3rd, Rubash HE. Periprosthetic fractures of the femur after total knee arthroplasty. A literature review and treatment algorithm. Clin Orthop Relat Res. 1991;(271):13542.

16. Moran MC, Brick GW, Sledge CB, Dysart SH, Chien EP. Supracondylar femoral fracture following total knee arthroplasty. Clin Orthop Relat Res. 1996;(324):196-209.

17. Healy WL, Siliski JM, Incavo SJ. Operative treatment of distal femoral fractures proximal to total knee replacements. J Bone Joint Surg Am. 1993;75:27-34.

18. Frigg R, Appenzeller A, Christensen R, Frenk A, Gilbert S, Schavan R. The development of the distal femur Less Invasive Stabilization System (LISS). Injury. 2001;32 Suppl 3:SC24-31.

19. Gurava Reddy V, Krishna Mootha A, Chiranjeevi T, Kantesaria P, Kumar Ramireddy V, Reddy D. Bilateral symmetrical periprosthetic (mirror) fractures of knee fixed with dual plating technique. Int J Surg Case Rep. 2011;2:175-7.

20. Zlowodzki M, Williamson S, Cole PA, Zardiackas LD, Kregor PJ. Biomechanical evaluation of the less invasive stabilization system, angled blade plate, and retrograde intramedullary nail for the internal fixation of distal femur fractures. J Orthop Trauma. 2004;18:494-502.

21. Kregor PJ, Hughes JL, Cole PA. Fixation of distal femoral fractures above total knee arthroplasty utilizing the Less Invasive Stabilization System (L.I.S.S.). Injury. 2001;32 Suppl 3: SC64-75.

22. Ruchholtz S, Tomas J, Gebhard F, Larsen MS. Periprosthetic fractures around the knee-the best way of treatment. Eur Orthop Traumatol. 2013;4:93-102.

23. Murrell GA, Nunley JA. Interlocked supracondylar intramedullary nails for supracondylar fractures after total knee arthroplasty: a new treatment method. J Arthroplasty. 1995; 10:37-42.

24. Lee SS, Lim SJ, Moon YW, Seo JG. Outcomes of long retrograde intramedullary nailing for periprosthetic supracondylar femoral fractures following total knee arthroplasty. Arch Orthop Trauma Surg. 2014;134:47-52.

25. Pelfort X, Torres-Claramunt R, Hinarejos P, Leal J, GilGonzalez S, Puig L. Extension malunion of the femoral component after retrograde nailing: no sequelae at 6 years. J Orthop Trauma. 2013;27:158-61.

26. Jabczenski FF, Crawford M. Retrograde intramedullary nailing of supracondylar femur fractures above total knee ar- throplasty: a preliminary report of four cases. J Arthroplasty. 1995;10:95-101.

27. Maniar RN, Umlas ME, Rodriguez JA, Ranawat CS. Supracondylar femoral fracture above a PFC posterior cruciatesubstituting total knee arthroplasty treated with supracondylar nailing: a unique technical problem. J Arthroplasty. 1996; 11:637-9.

28. Freedman EL, Hak DJ, Johnson EE, Eckardt JJ. Total knee replacement including a modular distal femoral component in elderly patients with acute fracture or nonunion. J Orthop Trauma. 1995;9:231-7.

29. Rand JA, Coventry MB. Stress fractures after total knee arthroplasty. J Bone Joint Surg Am. 1980;62:226-33.

30. Felix NA, Stuart MJ, Hanssen AD. Periprosthetic fractures of the tibia associated with total knee arthroplasty. Clin Orthop Relat Res. 1997;(345):113-24.

31. Alden KJ, Duncan WH, Trousdale RT, Pagnano MW, Haidukewych GJ. Intraoperative fracture during primary total knee arthroplasty. Clin Orthop Relat Res. 2010;468:90-5.

32. Ritter MA, Carr K, Keating EM, Faris PM, Meding JB. Tibial shaft fracture following tibial tubercle osteotomy. J Arthroplasty. 1996;11:117-9.

33. Lotke PA, Ecker ML. Influence of positioning of prosthesis in total knee replacement. J Bone Joint Surg Am. 1977;59:779.

34. Hanssen AD, Stuart MJ. Treatment of periprosthetic tibial fractures. Clin Orthop Relat Res. 2000;(380):91-8.

35. Goldberg VM, Figgie HE 3rd, Inglis AE, Figgie MP, Sobel M, Kelly M, Kraay M. Patellar fracture type and prognosis in condylar total knee arthroplasty. Clin Orthop Relat Res. 1988;(236):115-22.

36. Windsor RE, Scuderi GR, Insall JN. Patellar fractures in total knee arthroplasty. J Arthroplasty. 1989;4 Suppl:S63-7.

37. Ortiguera CJ, Berry DJ. Patellar fracture after total knee arthroplasty. J Bone Joint Surg Am. 2002;84:532-40.

38. Huberti HH, Hayes WC. Patellofemoral contact pressures. The influence of q-angle and tendofemoral contact. J Bone Joint Surg Am. 1984;66:715-24.

39. Wetzner SM, Bezreh JS, Scott RD, Bierbaum BE, Newberg AH. Bone scanning in the assessment of patellar viability following knee replacement. Clin Orthop Relat Res. 1985;(199): 215-9.

40. Goldstein SA, Coale E, Weiss AP, Grossnickle M, Meller B, Matthews LS. Patellar surface strain. J Orthop Res. 1986;4: $372-7$. 